\title{
Entanglement of fields in coupled-cavities: effects of pumping and fluctuations
}

\author{
S. Sivakumar \\ Materials Physics Division \\ Indira Gandhi Centre for Atomic Research \\ Kalpakkam 603102 INDIA \\ Email: siva@igcar.gov.in
}

December 15, 2018

\begin{abstract}
A system of two coupled cavities is studied in the context of bipartite, continuous variable entanglement. One of the cavities is pumped by an external classical source that is coupled quadratically, to the cavity field. Dynamics of entanglement, quantified by covariance measure [Dodonov et al, Phys. Lett A 296, (2002) 73], in the presence of cavity-cavity coupling and external pumping is investigated. The importance of tailoring the coupling between the cavities is brought out by studying the effects of pump fluctuations on the entanglement.
\end{abstract}

PACS: 42.50.Pq, 03.67.Bg, 03.67.Mn

Keywords: Entanglement, cavity QED, continuous variable, binomial states 


\section{Introduction}

Quantum information processing requires entangled states [1, 2, Many physical systems such as Josephson devices, trapped ions, NMR, have been considered for quantum information processing. All these system are endowed with finite number of states for manipulation. Of late, systems which are equipped with infinite dimensional Hilbert spaces have been analyzed in the context of teleportation, computation, etc [3, 4, 5, 6, 7]. Nonlinear optical processes, cavities in photonic bandgap materials are capable of producing states of light that are useful in quantum information processing. A notable feature of bosonic modes is their robustness in retaining their entanglement in spite of thermal noise [8]. Also, experiments with entangled photon states are of significance in testing the foundational aspects of quantum theory $[9]$.

Microcavities have been extensively employed in generating atomic qubits, engineering of quantum states, creating polarization entangled photonic states to do proof-of-principle tests such as verification of Bell-type inequalities, entangling atoms and/or photons [10, 11, 12], studying the phenomenon of decoherence [13, etc. More interestingly, coupled cavities have been thought of as a conduit to transfer and engineer entanglement between distant atomic qubits [14], which would be required for distributed quantum computing. Cavities containing atoms (either positioned or on-flight) have been used in the previously mentioned contexts. It is possible to use suitably prepared atoms to modify the cavity field so as to make the later useful in applications such as photonic qubits[15, 16], optical Josephson interferometers [17, etc.

In this work, a system of two coupled single-mode cavities is considered. The cavities are coupled by evanescent fields. Hence, the coupling strength can be tailored by the proximity of the cavities and the permittivity of the cavity walls. The field modes of the two cavities form a bipartite, bosonic, continuous variable (CV) system. Because of the coupling, the modes of the two cavities can get entangled. In addition to the inter-cavity coupling, one of the cavities is externally pumped. The results presented here pertain to the case of treating the pumping field classically.

The Hamiltonian of the system under consideration is

$$
\hat{H}=\omega\left(\hat{a}^{\dagger} \hat{a}+\hat{b}^{\dagger} \hat{b}\right)+\lambda\left(\hat{a}^{\dagger} \hat{b}+\hat{a} \hat{b}^{\dagger}\right)+\epsilon \hat{a}^{\dagger 2}+\epsilon^{*} \hat{a}^{2} .
$$

The operators $\hat{a}$ and $\hat{a}^{\dagger}$ are respectively the annihilation- and creation- op- 
erators of the mode ( $a$-mode, hereafter) in the cavity which is externally pumped. Similarly, $\hat{b}$ and $\hat{b}^{\dagger}$ are the relevant operators for the mode $(b$ mode, hereafter) in the other cavity. The strength of the coupling between the cavities is characterized by the parameter $\lambda$. The magnitude of this parameter is related to the hopping strength of photons to hop between the cavities. The term $\epsilon \hat{a}^{\dagger 2}+\epsilon^{*} \hat{a}^{2}$ corresponds to the contribution to the Hamiltonian from the interaction between the external pumping and the $a$ mode. This interaction, for instance, could be achieved by an active medium within the cavity. The magnitude of this interaction strength between the $a$-mode and the classical driving field is given by the parameter $\epsilon$ which incorporates the effects of the active medium and the external pumping. A similar approach is adopted to explain the maser action which includes many external modes 18. The external classical field affects entanglement as it delivers energy into the pumped cavity which supports the $a$-mode. However, whether the entanglement is enhanced or decreased depends on the coupling $(\lambda)$ between the cavities. Firstly, the dynamics in the absence of the external pumping is studied. These results are useful to understand the effects of pumping.

\section{Dynamics of fields: without pumping}

The Hamiltonian to study the dynamics in the absence of external driving corresponds to setting $\epsilon=0$ in $\hat{H}$. In that case, the Hamiltonian evolution is easily obtained since the commutator $\left[\hat{a}^{\dagger} \hat{a}+\hat{b}^{\dagger} \hat{b}, \hat{a}^{\dagger} \hat{b}+\hat{a} \hat{b}^{\dagger}\right]$ vanishes. This implies that the evolution operator $\exp (-i t \hat{H})$ can be factored as $\exp \left(-i t\left(\hat{a}^{\dagger} \hat{b}+\hat{a} \hat{b}^{\dagger}\right)\right) \exp \left(-i t\left(\hat{a}^{\dagger} \hat{a}+\hat{b}^{\dagger} \hat{b}\right)\right)$. This type of Hamiltonian is known in other contexts such as the symmetric beam splitter 19, the parametric conversion in a medium with oscillatory dielectric constant 20] and has recently been used to examine intrinsic phase coherence in a laser [21. The bilinear coupling $\lambda\left(\hat{a}^{\dagger} \hat{b}+\hat{a} \hat{b}^{\dagger}\right)$, between the modes cannot entangle them during evolution if both the modes are in classical states initially 22, 23]; for instance, the $a$-mode in a coherent state and the $b$-mode in vacuum state. To generate an entangled state from a product state, during evolution in the presence of bilinear coupling, at least one of the modes has to be in a nonclassical state. So, it is meaningful to assume the initial state of the cavities to be a number state. Since all number states, except the vacuum, are nonclassical, the bilinear coupling may entangle the $a$ - and $b$ - fields. It is experimentally possible to prepare the cavities such that $a$-field is a Fock 
state, say $|N\rangle$, and the $b$-field to be vacuum state.

Another consequence of the vanishing commutator $\left[\hat{a}^{\dagger} \hat{a}+\hat{b}^{\dagger} \hat{b}, \hat{a}^{\dagger} \hat{b}+\hat{a} \hat{b}^{\dagger}\right]=$ 0 , is the existence of nontrivial, irreducible, invariant subspaces. Each invariant subspace is the span of the states of the form $\{|m, N-m\rangle\}$, where $m$ runs from zero to $N$. The state $|m, N-m\rangle$ of the modes in the cavities represents the situation in which the $a$-mode is in the number state $|m\rangle$ and the $b$-mode is in $|N-m\rangle$. There is an invariant subspace associated with each value of $N$, an eigenvalue of the total number operator $\hat{a}^{\dagger} \hat{a}+\hat{b}^{\dagger} \hat{b}$. All the states in an invariant subspace are the eigenstates of the total number operator. Further, the invariant subspaces corresponding to different total quantum numbers are disjoint. Taking the initial state as $|N, 0\rangle$, the time-evolved state $|\psi(t)\rangle=\exp (-i \hat{H} t)|N, 0\rangle$ is

$$
|\psi(t)\rangle=\exp (-i N \omega t) \cos ^{N}(\lambda t) \sum_{n=0}^{N}\left[\frac{N !}{n !(N-n) !}\right]^{\frac{1}{2}} \tan ^{n}(\lambda t)|N-n, n\rangle .
$$

This state belongs to the invariant subspace corresponding to a total quantum number $N$ which contains the initial state $|N, 0\rangle$. An interesting feature is that the states of the form $|m, N-m\rangle$ are the only product states in the invariant subspace. All the other states in a given invariant subspace are entangled [24]. The coefficients in the Fock state expansion are the binomial coefficients and the states defined in Eq. 2 are referred as two-mode binomial states 25].

In this work, the covariance criterion is adopted as the measure of entanglement [26]. It is expressed as

$$
Y=\left[\frac{\left|\overline{\hat{a} \hat{b}^{\dagger}}\right|^{2}+|\overline{\hat{a} \hat{b}}|^{2}}{2\left(\overline{\hat{a}^{\dagger} \hat{a}}+\frac{1}{2}\right)\left(\overline{\hat{b}^{\dagger} \hat{b}}+\frac{1}{2}\right)}\right]^{\frac{1}{2}} .
$$

The bar is used to indicate covariance, for example, $\overline{\hat{a} \hat{b}^{\dagger}}=\left\langle\hat{a} \hat{b}^{\dagger}\right\rangle-\langle\hat{a}\rangle\left\langle\hat{b}^{\dagger}\right\rangle$, where $\langle\cdots\rangle$ stands for quantum mechanical expectation value of the relevant operator. The quantity $Y$ is non-negative and less than unity. For product states, the value of $Y$ is zero. Nonzero values of $Y$ implies the state is entangled. But there exist entangled states for which $Y$ is zero and hence the criterion is not universal. Nevertheless, it is easy to compute and useful in identifying and quantifying entanglement when it assumes nonzero values. If the initial state is of the form $|N, 0\rangle$, the covariance parameter of the 
evolved state given in Eq. 2 is

$$
Y=\frac{N|\sin (2 \lambda t)|}{2\left[2\left(N \cos ^{2}(\lambda t)+.5\right)\left(N \sin ^{2}(\lambda t)+.5\right)\right]^{1 / 2}} .
$$

With nonzero coupling, the maximum value that $Y$ attains during evolution is $N / \sqrt{2}(N+1)$ and it is attained whenever $\lambda t$ is an odd integral multiple of $\pi / 4$. It is clear that the peak value of $Y$ attained during evolution increases with $N$ and approaches $1 / \sqrt{2}$ asymptotically as $N$ becomes large. Further, the peak value depends on the total quantum number $N$ and not on the fieldfield coupling constant $\lambda$. In Fig. 1 the variation of $Y$ with time is given for different values of the total quantum number, which is the eigenvalue of the operator $\hat{a}^{\dagger} \hat{a}+\hat{b}^{\dagger} \hat{b}$. The time of evolution is measured in the units of $\pi / \lambda$, which is the temporal periodicity of pumping-free evolution. In all the subsequent discussions, whether the evolution is periodic or not, the time is always expressed in the units of $\pi / \lambda$.

The universal measure of entanglement for bipartite pure states [27] is the von Neumann entropy $S$ defined as

$$
S=-\operatorname{Tr}\left[\hat{\rho}_{a} \log _{2} \hat{\rho}_{a}\right],
$$

where $\operatorname{Tr}$ stands for trace and $\hat{\rho}_{a}$ is the reduced density matrix for the $a$ mode field. It is instructive to study the behaviour of von Neumann entropy (S) vis-a-vis $Y$ in the present case. For the states defined in Eq. 2, the reduced density matrix $\hat{\rho}_{a}$ for the $a$-field is

$$
\hat{\rho}_{a}=\cos ^{2 N}(\lambda t) \sum_{n=0}^{N} \frac{N !}{n !(N-n) !} \tan ^{2 n}(\lambda t)|N-n\rangle\langle N-n| .
$$

Denoting the coefficient of $|N-n\rangle\langle N-n|$ in $\hat{\rho}_{a}$ by $c_{n}$, the expression for the entropy is $S=-2 \sum_{n=0}^{N}\left|c_{n}\right|^{2} \log _{2}\left|c_{n}\right|$. In Fig. 2, the variation of $S$ and $Y$ are shown as functions of time taking the initial state to be $|5,0\rangle$. The two measures exhibit very similar features. When $S$ is nonzero, the covariance measure $Y$ is nonzero too, indicating that it is a good criterion for the kind of entangled states given in Eq. 2, The peak in the entropy occurs when the two modes have nearly equal number of photons, that is, $\left\langle\hat{a}^{\dagger} \hat{a}\right\rangle \approx\left\langle\hat{b}^{\dagger} \hat{b}\right\rangle$. Hence, entropy peaks correspond to minima in the difference of the photon numbers of the two modes. The photon number in the $a$-mode is $N \cos ^{2}(\lambda t)$ and in the $b$-mode is $N \sin ^{2}(\lambda t)$. Therefore, $\left\langle\hat{a}^{\dagger} \hat{a}\right\rangle$ equals $\left\langle\hat{b}^{\dagger} \hat{b}\right\rangle$ whenever $\lambda t$ is an odd multiple of $\pi / 4$. The reason for the increase in the entanglement as the total number of photons increases is readily inferred from the expression 
for entropy $S$ given after Eq, 6 . The entropy $S$ is maximum, subject to the constraint that $\operatorname{Tr} \rho_{a}=1$, when all $\left|c_{n}\right|$ are equal. There are $N+1$ terms in the expression for $S$ and the maximum $S$ is $\log _{2}(N+1)$ which increases with $N$.

\section{$3 \quad$ Effect of external pumping}

The evanescent coupling allows photons to hop from one cavity to the other. This allows a redistribution of energy between the cavities. Since the $a$-mode is subjected to external pumping, the energy of the pump field is fed to the $a$-mode which, in turn, is used to energize the $b$-field. The pump field is treated classically and hence the driving term in the Hamiltonian is a function of the operators of the $a$-mode. For weak couplings, it is reasonable to assume the function to be linear in $\hat{a}^{\dagger}$ and $\hat{a}$. Classically, this amounts to an interaction proportional to the product of the field strength of the $a$ mode and the amplitude of the driving field. If the coupling is stronger, the function must involve higher order terms involving the creation and the annihilation operators. In what follows, the effects of quadratic coupling on the entanglement between the cavities are discussed. As a remark, it is noted that if the pumping is linear, that is, the driving term is $\epsilon \hat{a}^{\dagger}+\epsilon^{*} \hat{a}$, there is no effect on the value of the covariance parameter $Y$. There is indeed a change in the dynamics and, consequently the entanglement is affected. But the dynamics of the covariance criterion is insensitive to such modifications, a reminiscence of the fact that the covariance criterion is not universal. The quadratic coupling, however, leads to significant changes in the dynamics of $Y$.

The Heisenberg equations of motion for the operators of the two mode are

$$
i \frac{d}{d t}\left(\begin{array}{c}
\hat{a} \\
\hat{b} \\
\hat{a}^{\dagger} \\
\hat{b}^{\dagger}
\end{array}\right)_{t}=\left[\begin{array}{cccc}
\omega & \lambda & 2 \epsilon & 0 \\
\lambda & \omega & 0 & 0 \\
-2 \epsilon^{*} & 0 & -\omega & -\lambda \\
0 & 0 & -\lambda & -\omega
\end{array}\right]\left(\begin{array}{c}
\hat{a} \\
\hat{b} \\
\hat{a}^{\dagger} \\
\hat{b}^{\dagger}
\end{array}\right)_{t} .
$$

The equations imply that the evolution of the operators of the two modes are coupled. The coefficient matrix has no explicit time-dependence and 
hence the equations are easily solved to obtain

$$
\left(\begin{array}{c}
\hat{a} \\
\hat{b} \\
\hat{a}^{\dagger} \\
\hat{b}^{\dagger}
\end{array}\right)_{t}=\exp [-i t \hat{M}]\left(\begin{array}{c}
\hat{a} \\
\hat{b} \\
\hat{a}^{\dagger} \\
\hat{b}^{\dagger}
\end{array}\right)_{0}
$$

where $\hat{M}$ represents the coefficient matrix in Eq. 7 ,

The operator $\exp [-i t \hat{M}]$ can be expressed in terms of lower powers of $\hat{M}$ using Cayley-Hamilton theorem[28]. Let the four eigenvalues of $\hat{M}$ be denoted by $\alpha, \beta, \gamma$ and $\delta$. Defining $A=\omega^{2}+\lambda^{2}-2 \epsilon^{2}$ and $B=\sqrt{\omega^{2} \lambda^{2}-\lambda^{2} \epsilon^{2}+\epsilon^{4}}$, the eigenvalues are

$$
\begin{gathered}
\alpha=-\beta=\sqrt{A-2 B}, \\
\gamma=-\delta=\sqrt{A+2 B} .
\end{gathered}
$$

These are the eigenvalues if $\epsilon$ is real and they are distinct if the determinant of $\hat{M}$ is nonzero, which is always the case for the typical values of the parameters assumed. Now, applying Cayley-Hamilton theorem,

$$
\begin{aligned}
4 B \exp [-i t \hat{M}]= & i\left(\frac{\sin \alpha t}{\alpha}-\frac{\sin \gamma t}{\gamma}\right) \hat{M}^{3} \\
& +(\cos \gamma t-\cos \alpha t) \hat{M}^{2}-i\left(\frac{\alpha^{2} \sin \gamma t}{\gamma}-\frac{\gamma^{2} \sin \alpha t}{\alpha}\right) \hat{M} \\
& -\left(\gamma^{2} \cos \alpha t-\alpha^{2} \cos \gamma t\right) I_{4}
\end{aligned}
$$

where $I_{4}$ is the $4 \times 4$ identity matrix.

On using the matrix exponential, refer Eq. 11, in the solution given in Eq. 8, the expectation values of time-evolved operators are expressed in terms of their initial expectation values. Let the time- dependent coefficients of $\hat{M}^{3}, \hat{M}^{2}, \hat{M}$ and $I_{4}$ in the expression for $\exp [-i t \hat{M}]$ given in Eq. 11, be $C_{3}, C_{2}, C_{1}$ and $C_{0}$ respectively. Further, define

$$
\begin{aligned}
U_{\omega, \lambda} & =C_{0}+\omega C_{1}+\left(A-2 \epsilon^{2}\right) C_{2}+\omega\left(2 \lambda^{2}-2 \epsilon^{2}+A\right) C_{3}, \\
V_{\omega, \lambda} & =\lambda C_{1}+2 \lambda \omega C_{2}+\lambda\left(2 \omega^{2}-2 \epsilon^{2}+A\right) C_{3} \\
W_{\omega, \lambda} & =2 \epsilon C_{1}+2 \epsilon\left(\lambda^{2}+A\right) C_{3} \\
X_{\omega, \lambda} & =C_{0}+\omega C_{1}+\left(\lambda^{2}+\omega^{2}\right) C_{2}+\omega\left(3 \lambda^{2}+\omega^{2}\right) C_{3} \\
Y_{\omega, \lambda} & =2 \epsilon\left(\lambda C_{2}+\omega C_{3}\right) \\
Z_{\omega, \lambda} & =-2 \lambda^{2} \epsilon C_{3} .
\end{aligned}
$$


If the initial state of the two cavities is $|N, 0\rangle$, the relevant quantities to compute $Y$ are

$$
\begin{aligned}
\overline{\hat{a} \hat{b}} & =(1+N) X_{\omega, \lambda} Y_{\omega, \lambda}+N W_{\omega, \lambda} V_{\omega, \lambda}+V_{\omega, \lambda} Z_{\omega, \lambda}, \\
\overline{\hat{a} \hat{b}^{\dagger}} & =(1+N) U_{\omega, \lambda} V_{-\omega,-\lambda}+N W_{\omega, \lambda} Y_{-\omega, \lambda}+V_{\omega, \lambda} X_{-\omega,-\lambda}, \\
\overline{\hat{a}^{\dagger} \hat{a}} & ==(1+N) U_{\omega, \lambda} U_{-\omega,-\lambda}-N W_{\omega, \lambda}^{2}+V_{\omega, \lambda} V_{-\omega,-\lambda}-1, \\
\overline{\hat{b}^{\dagger} \hat{b}} & ==(1+N) V_{\omega, \lambda} V_{-\omega,-\lambda}+N Y_{\omega, \lambda} Y_{-\omega, \lambda}+X_{\omega, \lambda} X_{-\omega,-\lambda}-1 .
\end{aligned}
$$

Using the expressions in Eqs. 12,15] for the various bilinear combinations of the creation- and annihilation- operators of the two cavity modes, the covariance criterion of entanglement is computed. In Fig. 3 the evolution of $Y$ with time is shown for various combinations of $\lambda$ and $\epsilon$. When both $\lambda$ and $\epsilon$ are chosen to be 0.1, the entanglement measure $Y$ builds up to 0.6. The occurrence of peak values in $Y$ is corroborated with the occurrence of minimum in the difference between the photon numbers of the two modes. The variation of the ratio of the mean photon number difference $\left|\left\langle\hat{a}^{\dagger} \hat{a}-\hat{b}^{\dagger} \hat{b}\right\rangle\right|$ to the total number of photons $\left|\left\langle\hat{a}^{\dagger} \hat{a}+\hat{b}^{\dagger} \hat{b}\right\rangle\right|$ is shown in Fig. 4. If the ratio is unity, the photon number of one of the modes is zero. If the ratio is zero, the two modes have equal number of photons. The evolution of the ratio is shown for three different combinations of $\lambda$ abd $\epsilon$. If the cavity-cavity coupling $\lambda$ is sufficiently strong that the energy increase due to the external pumping gets distributed to both the cavities, the difference in the average photon numbers in the two cavities can become zero, as in the case of $\lambda$ and $\epsilon$ both being 0.1 . If $\lambda$ is small, the energy transfer between the cavities is ineffective and the photon number difference is large, for instance, when $\lambda=0.001$ and $\epsilon=0.1$. To illustrate the effect of larger $\lambda$, the evolution of the ratio if $\lambda=0.005$ and $\epsilon=0.1$ is shown in Fig. 4. In this case, the difference becomes smaller than the $\epsilon=0.001$ case; however, the coupling is still not strong enough for efficient energy exchange between the cavities to make the difference in the photon number to become zero.

The occurrences of vanishing photon-number difference and the peak in $Y$ happen at same instants, seen by comparing Figs. 3 and 4 . When the pump-field coupling is large, the $a$-mode gets energized at a faster rate. If the coupling $\lambda$ is such that the exchange of energy between the cavities is effective, the $a$-field and the $b$ - field attain nearly equal energies so that the difference of their photon numbers becomes small at various instants. This, as in the case of pump-free evolution, is correlated with the occurrence of 
entanglement peaks. Similarly, if both $\lambda$ and $\epsilon$ are equal but smaller, chosen to be 0.001 for the purpose of discussion, the slow rate of energy build up in the $a$-mode is matched by the flow of energy between the modes. Hence, in this case too the energies of the two modes can be nearly equal during evolution. At such instants, the entanglement becomes higher.

If $(\lambda<<\epsilon)$, for instance, $\lambda=0.001$ and $\epsilon=0.1$, the external pumping primarily enhances the energy of the $a$-field as there is no effective flow of energy to the $b$-field because of low value of the coupling between the cavities. This increases the energy difference between the modes. The evolution of $Y$ in this case is shown in Fig. 3. Compared to the pump-free evolution of the initial state $|5,0\rangle$ shown in Fig. 1, the maximum entanglement attained is smaller as the energy difference between the modes is larger. The $b$-mode is initially in vacuum state. Due to the weak coupling between the cavities, the $b$-mode remains in a state in which only the vacuum state and a few number states are present. Therefore, the entanglement becomes smaller. The entanglement attains a peak whenever the energy difference is minimum. Thus, generation of large entanglement requires that energy flows into the $b$-field, which, in turn, requires a strong interaction between the cavities.

In the opposite limit $\lambda>>$, for instance $\lambda=0.1$ and $\epsilon=0.001$, the total energy of the system does not increase much as pumping is weak. The dynamics is very similar to that in the case when there is no pumping. The effective coupling between the modes leads to nearly equal energies in the modes during evolution. Whenever the mean photon numbers of the modes match, the entanglement attains a peak. The evolution profile of $Y$ is not distinctly seen in Fig. 3 as it merges with the profile corresponding to $\lambda=0.001$ and $\epsilon=0.001$.

\section{Effect of pump fluctuations}

The maximum value that $Y$ attains during evolution is dependent on the initial photon number $N$, the coupling $\lambda$ and the pump-field interaction strength $\epsilon$. In Fig. 5 the maximum value of $Y$ attained during evolution is shown as a function of $\epsilon$. Results corresponding to two different values of $\lambda$ are given taking the initial state to be $|5,0\rangle$. It is seen that the peak value increases with $\epsilon$ if $\lambda$ is 0.1 whereas it decreases drastically if $\lambda=0.001$. 
If $\lambda=0.1$, the peak value of $Y$ is close to 0.6 , for small values of $\epsilon$. This is nearly the value that is attained in the absence of pumping since the pumping is weak. As the value of $\epsilon$ increases beyond 0.05 , the peak value increases. For sufficiently large $\epsilon$, say, $\approx 0.4$, the peak value corresponds to the maximum attainable in pumping-free case and initial $N$ as large as 50. This is understandable as large pumping leads to large energy input. Since $\lambda=0.1$ is sufficient enough for energy transfer between the modes, both the modes will have more number of Fock states in their superposition. Hence, a large entanglement is possible. If $\lambda=0.001$, a representative value for small $\lambda$, the coupling is not good enough for the energy to flow from the $a$-mode. Thus, the $b$-mode will have fewer number of Fock states in the superposition. Also, as $\epsilon$ increases, the difference in the photon number of the modes will become larger. So, there is reduction in the maximum entanglement between the modes as compared with the values in the absence of pumping. The dependence of peak $Y$ on $\epsilon$ is shown in Fig. 5 for two other values of $\lambda$. It is seen that as $\lambda$ becomes larger, the peak $Y$ during evolution is nearly constant over a larger range of $\epsilon$.

From Fig. 5, it is seen that if $\lambda$ is 0.1 , the maximum attainable $Y$ varies from 0.6 to 0.7 as $\epsilon$ increases from 0 to 0.5 . On the other hand, if $\lambda=0.001$ the maximum attainable $Y$ changes from 0.6 to 0.03 as $\epsilon$ varies from 0 to 0.5. The change in maximum $Y$ is larger for the smaller $\lambda$ implying a larger sensitivity to changes in $\epsilon$. This has important consequences. In any pumping scheme there are fluctuations in the driving amplitude. The effect of these fluctuations on the entanglement is decided by the sensitivity of the system. From the discussion it is expected that the dynamics should not differ much if $\lambda$ is large whereas perceptible changes are possible if $\lambda$ is small. To account for the driving field fluctuations, the coefficient $\epsilon$ is assumed to fluctuate about its mean value. The fluctuation is assumed to be Gaussian with mean equal to the value of assumed value of $\epsilon$ and variance to be one-tenth of the mean. When the mean value of $\epsilon$ is 0.001 , the fluctuations do not affect the entanglement. But at higher mean values, fluctuations in $\epsilon$ affect entanglement dynamics. Taking mean of $\epsilon$ to be 0.3 , the timeevolution of $Y$ is shown in Fig. 6 for $\lambda=0.001$ and 0.1 . The fluctuating driving term is taken to be piecewise constant function. The total evolution time is divided into 100 equal parts; in each part, the value of $\epsilon$ is chosen at random from the Gaussian distribution defined earlier. The evolution is tracked for ten different random trials in each case. The evolution of $Y$ corresponding to small $\lambda$, shown in (a) of Fig. 6, shows a visible spread in the evolution during different trials. In order to distinctly bring out the effects of fluctuations, the dynamics is continued for five units of scaled time. The 
entanglement itself is very small as the field-field coupling is small. But the fluctuations induce large relative changes in the entanglement. Thus, strong pumping and weak coupling between the cavities lead to large uncertainties in entanglement. With larger $\lambda$, refer (b) in Fig. 6, the spread is almost insignificant. Strong coupling between the modes reduces the effect of the driving-field fluctuations on the entanglement. As stressed earlier, sufficient coupling between the cavities allows for efficient flow of energy and the maximum value of $Y$ during evolution is also large. It is, therefore, possible to choose the cavity-cavity coupling so that the effects of fluctuations are minimized. In other words, smaller coupling between the cavities necessitates a more stable pumping.

\section{Summary}

Two coupled cavities generate entangled, bipartite, non-Gaussian states. In the absence of external pumping, the cavities exchange energy periodically. The entanglement attained during evolution increases with the number of photons present in the cavities initially. Inclusion of external pumping in one of the cavities amplifies the field in that cavity, which, in turn, energizes, via the evanescent coupling, the mode in the other cavity. This affects the entanglement between the modes. The covariance criterion of entanglement is insensitive to the driving amplitude if the driving is linear. However, with quadratic pumping, the states occurring during the evolution are such that the covariance criterion identifies entanglement. If the couping between cavities is weak, increasing the driving field amplitude decreases the peak entanglement attainable during evolution compared to the pump-free evolution. Therefore, it is important to tailor the pumping strength to match the cavity-cavity coupling. During evolution, maximum entanglement between the fields in the cavities occurs when the mean number of photons in the two cavities are equal.

For weak coupling between the cavities, the maximum attainable entanglement decreases rapidly as the pumping strength is increased. Though the entanglement increases for weak pumping, it begins to decrease for further increase of pumping strength. This is because of preferential increase in the energy of the pumped cavity leading to large difference in the mean number of photons of the two cavities. On the other hand, if the coupling between the cavities is stronger, the maximum entanglement attained during evolu-

tion increases for larger range of values of the pumping strength. Also, the 
change of entanglement with pumping strength is not as rapid as the change if the coupling between the cavities is weak. Consequently, the evolution of entanglement is less sensitive to fluctuations in the pumping if the cavities are coupled effectively.

\section{References}

[1] M. A. Nielsen, I. L. Chuang, Quantum Computation and Quantum Information, Cambridge University Press, Cambrdige, 2000.

[2] D. Esteve, J. -M. Raimond, J. Dalibard (Eds.), Quantum Entanglement and information processing, Les Houches Volume LXXIX, Elsevier B.V., The Netherlands, 2004.

[3] S L Braunstein, P van Loock, Rev. Mod. Phys. 77 (2005) 513-577.

[4] S. L. Braunstein, A. K. Pati (Eds.), Quantum Information with Continuous Variables, Kluwer Academic Publishers, The Netherlands, 2005.

[5] A. Furusawa et al, Science 282 (1998) 706-709.

[6] N. J. Cerf, G. Leuchs, E.S. Polzik (Eds.), Quantum Information with Continuous Variables of Atom and Light, Imperial College Press, London, 2007.

[7] G. Chen et al (Eds.), Quantum Devices Principles, Design, and Analysis, Chapman \& Hall, USA, 2007.

[8] E. Ciancio, P. Zanardi, Phys. Lett. A 360 (2006) 49-56.

[9] A. Aspect in: R. A. Bertlmann, A. Zeilinger (Eds.), Quantum [Un]speakables - From Bell to Quantum Information, Springer-Verlag, Berlin, 2002, pp.119-154.

[10] K. Vahala, Nature 224 (2003) 839-846.

[11] J. Raimond, M. Brune, S. Haroche, Rev. Mod. Phys. 73 (2001) 565-582.

[12] J. Cho, D. G. Angelakis, S. Bose, Phys. Rev. A 78 (2008) 022323-1-4.

[13] H. Mabuchi, A. C. Doherty, Science 298 (2002) 1372-1377.

[14] J. I. Cirac, P. Zoller, H. J. Kimble, H. Mabuchi, Phys. Rev. Lett. 78 (1997) 3221-3224. 
[15] K. M. Gheri, C. Saavedra, P. Torma, J. I. Cirac, P. Zoller, Phys. Rev. A 58 (1998) R2627-R2630.

[16] S. J. van Enk, J. I. Cirac, P. Zoller, Science 279 (1998) 205-208.

[17] D. Gerace, H. E. Tureci, A. Imamoglu, V. Giovannetti, R. Fazio, Nat. Phys. 5 (2009) 281-284.

[18] W. H. Louisell, Radiation and Noise in Quantum Electronics, McGrawHill, 1964, pp 272.

[19] G. C. Gerry, P. L. Knight, Introductory Quantum Optics, Cambridge University Press, Cambridge, 2004.

[20] W. Louisell, A. Yariv, A. Siegman, Phys. Rev. 124 (1961) 1646-1654.

[21] D T Pegg, Phys. Rev. A 79 (2009) 053837-1-8.

[22] M. S. Kim, W. Son, V. Buzek, P. L. Knight, Phys. Rev. A 65 (2002) 032323-1-7.

[23] Wang Xiang-bin, Phys. Rev. A 66 (2002) 024303-1-2.

[24] S. Sivakumar, J. Phys. B: At., Mol. and Opt. Phys. 42 (2009) 095502$1-10$

[25] D. Stoler, B. E. A. Saleh, M. C. Teich, Opt. Acta 32 (1985) 345-355.

[26] V. V. Dodonov, A. S. M. de Castro, S. S. Mizrahi, Phys. Lett. A 296 (2002) $73-81$.

[27] C. H. Bennett, H. J. Bernstein, S. Popescu, B. Schumacher, Phys. Rev. A 53 (1996) 2046-2052.

[28] T. M. Apostol, Calculus Vol. II, John Wiley, London, 2003. 


\section{Figure Captions}

Fig.1 Variation of $Y$ with time. The time-axis represents scaled-time defined as the ratio of time to $\pi / \lambda$. One unit of scaled-time corresponds to $\pi / \lambda$. The curves shown correspond to different total quantum numbers: $\mathrm{N}=1$ (dash), 5 (dot-dash), 10 (continuous) and 50 (dot). In all cases the coupling $\lambda$ is 0.1 .

Fig. 2 Variation of covariance measure $Y$ (continuous) and von Neumann entropy $S$ (dash) with scaled time. The initial state is $|5,0\rangle$ and $\lambda=0.1$.

Fig. 3 Comparison of evolution of $Y$ for weak and strong couplings when pumping is quadratic. Different plot types are used for different combinations of $\lambda$ and $\epsilon$ : dashed $-\lambda=0.001$ and $\epsilon=0.1$; continuous $-0.001,0.001$, beaded $-0.1,0.1$. The continuous curve hides the curve corresponding to the case: $\lambda=0.1$ and $\epsilon=0.001$. The initial state is $|5,0\rangle$.

Fig. 4 Ratio of $\left|\left\langle\hat{a}^{\dagger} \hat{a}-\hat{b}^{\dagger} \hat{b}\right\rangle\right|$ to $\left\langle\hat{a}^{\dagger} \hat{a}+\hat{b}^{\dagger} \hat{b}\right\rangle$ as a function of time. The curves shown correspond to $\lambda=0.001$ and $\epsilon=0.1$ (dash); 0.001, 0.001 (continuous), 0.1,0.005 (dots).

Fig. 5 Maximum value of $Y$ as a function of $\epsilon$. For weak coupling $\lambda=0.001$ (continuous)and for strong coupling $\lambda=0.05$ (dots). Additional curves correspond to $\lambda=0.005$ (dot-dash) and 0.01 (dash). The initial state is $|5,0\rangle$.

Fig. 6 Evolution of $Y$ in time under fluctuating driving field. The mean driving amplitude is 0.3. Two values of field-field couplings are assumed: $\lambda=0.001$ [figure (a)] and $\lambda=0.05$ [figure(b)]. Different evolution profiles correspond to different trials. In each of the figures, results of ten trial evolutions are given. The initial state is $|5,0\rangle$ for all the profiles. 


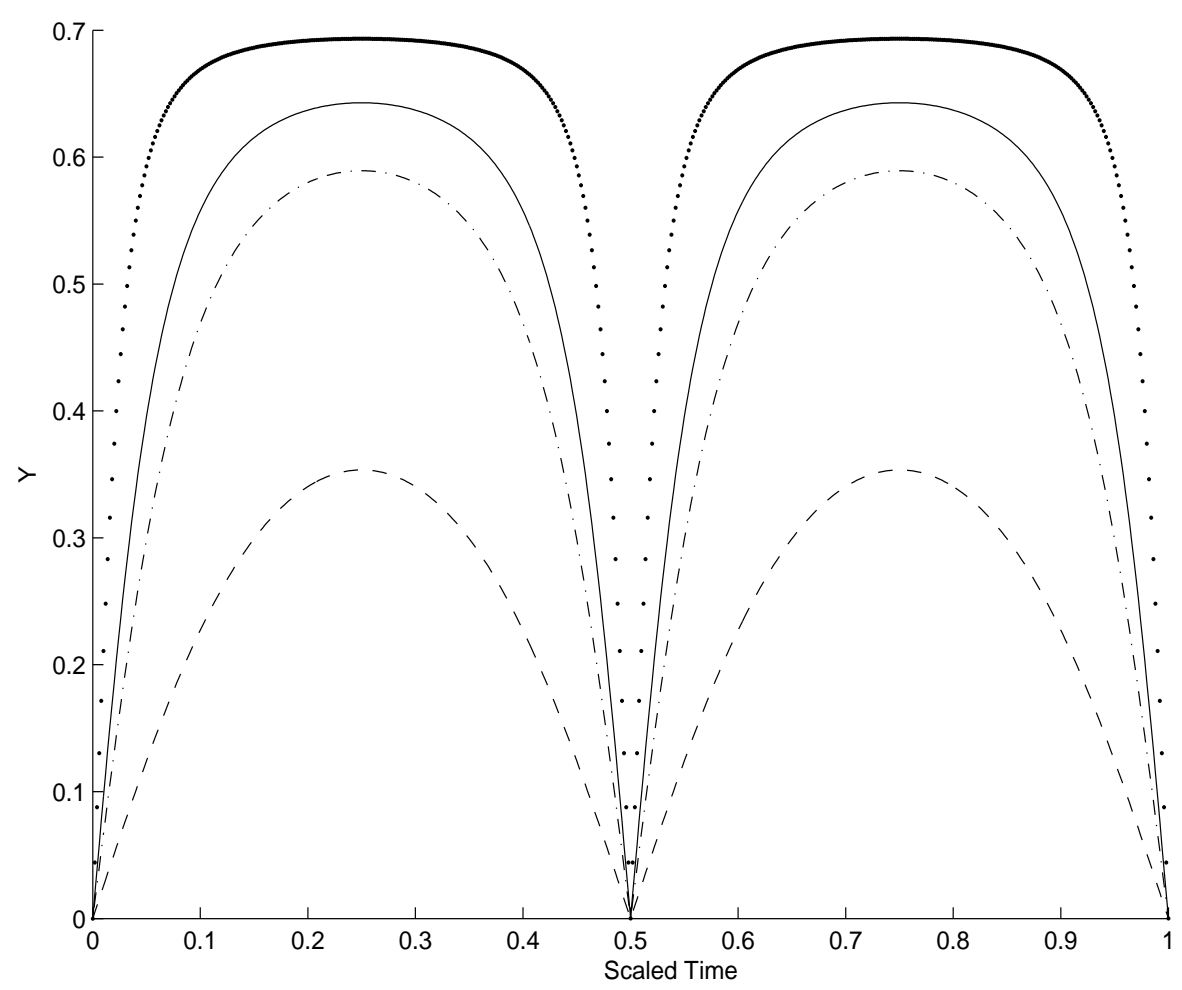

Figure 1: 


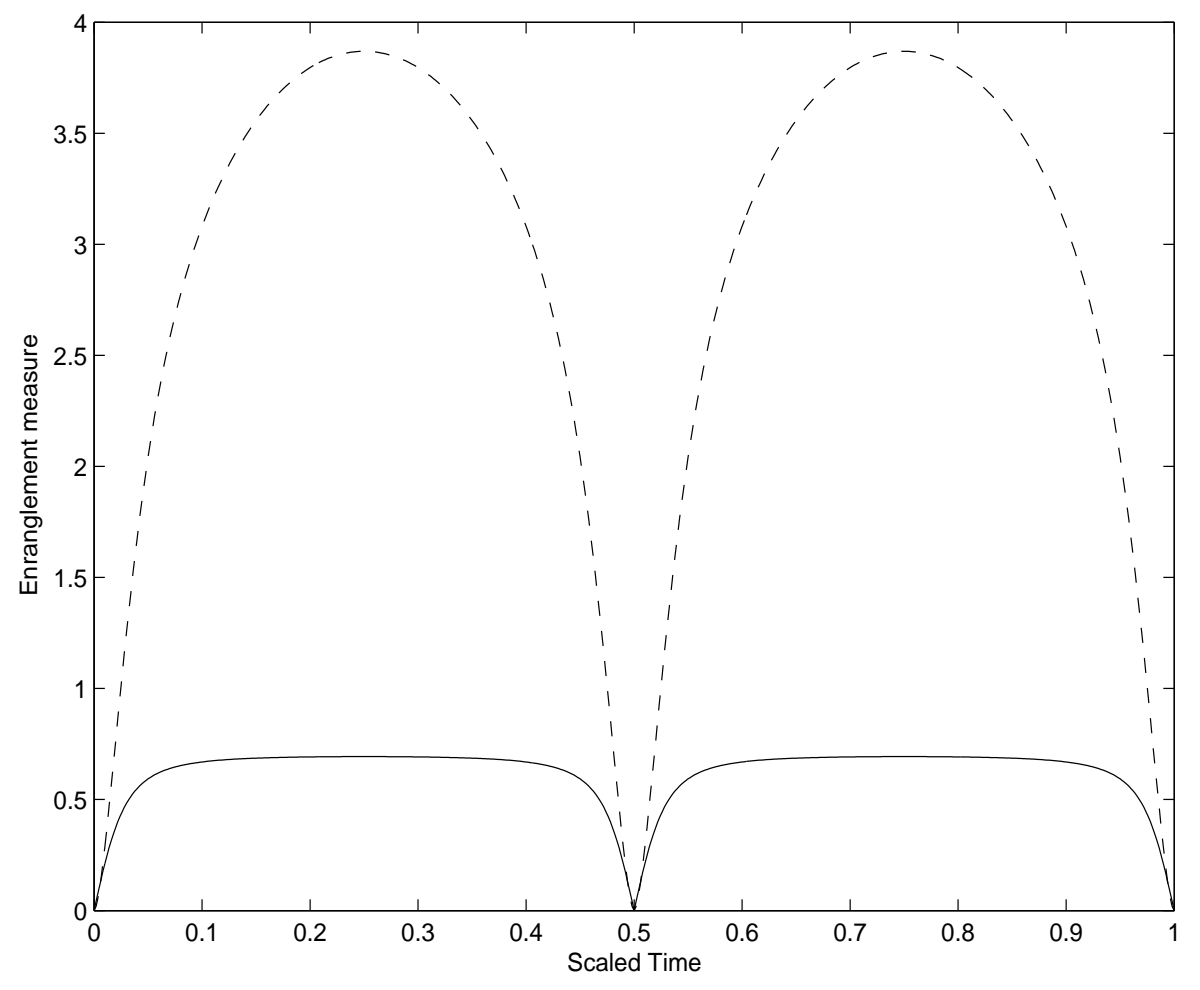

Figure 2: 


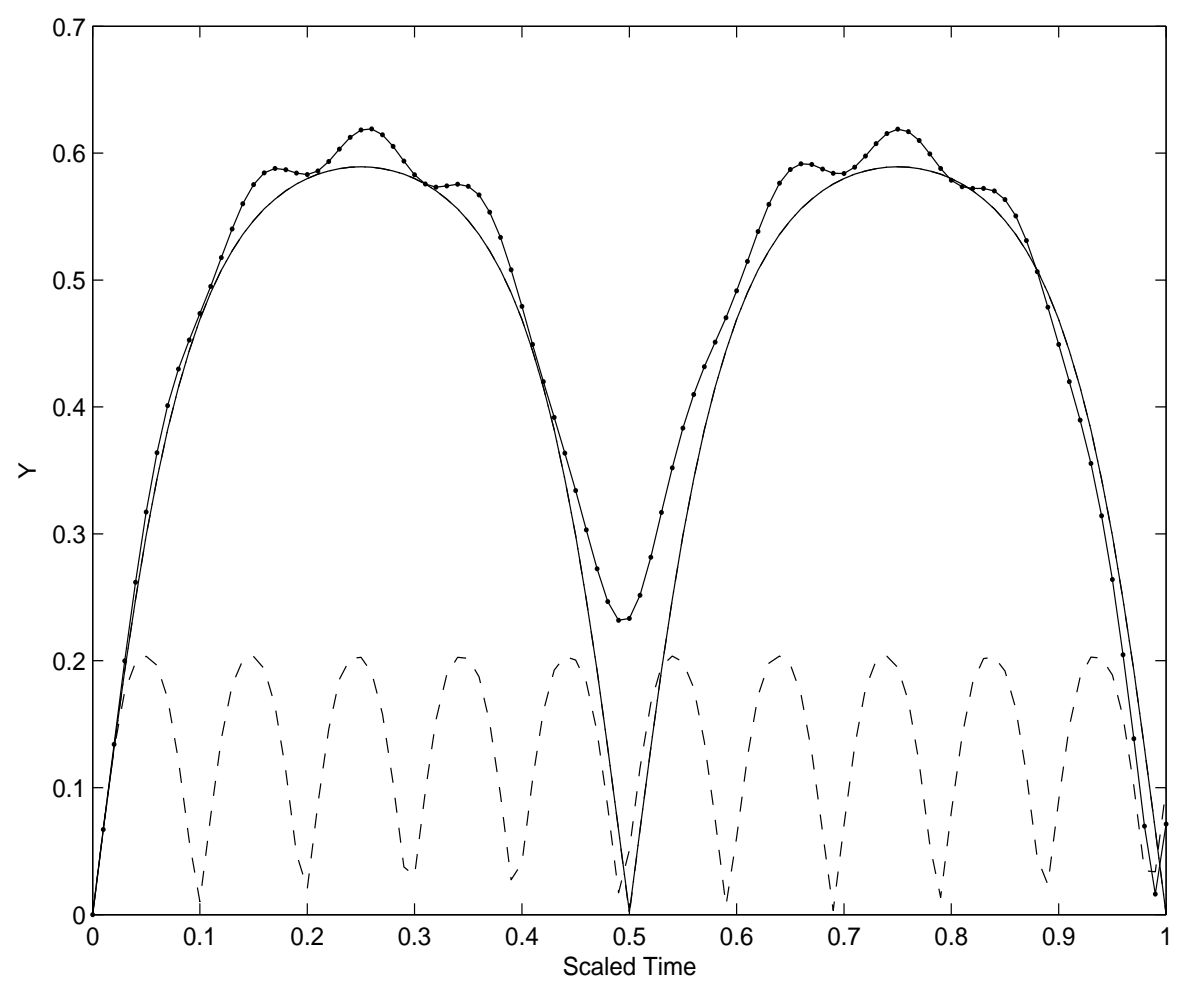

Figure 3: 


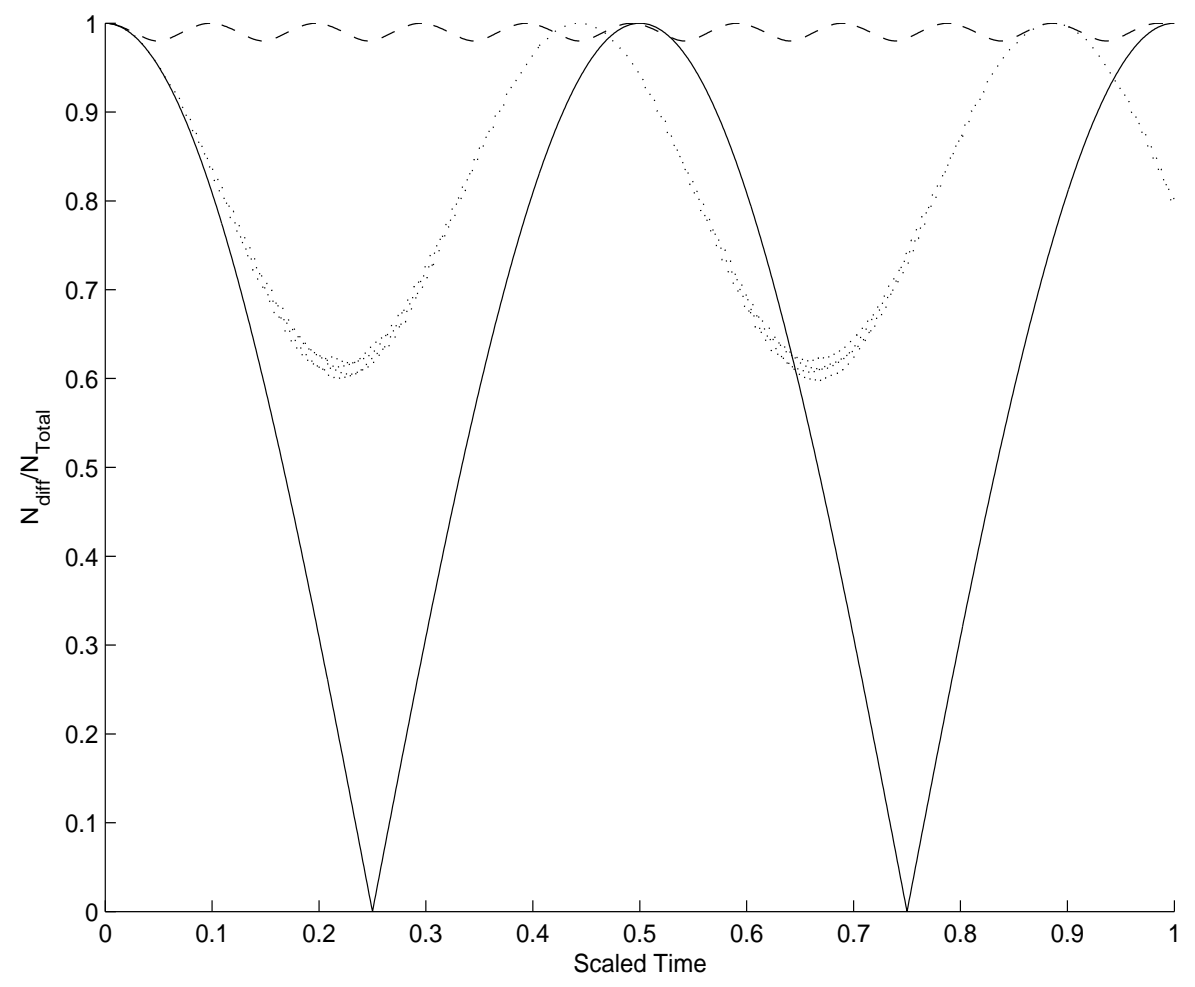

Figure 4: 


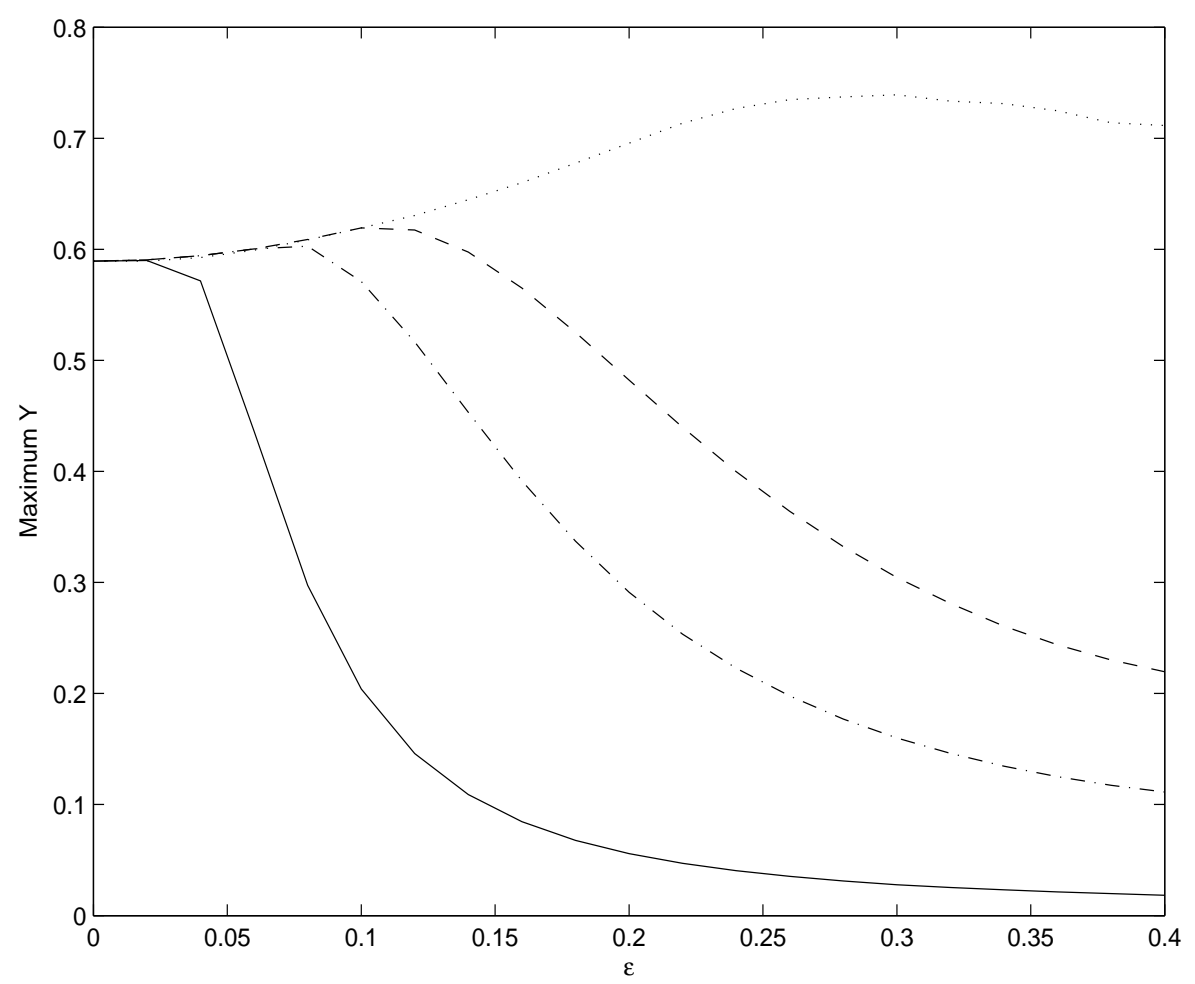

Figure 5: 

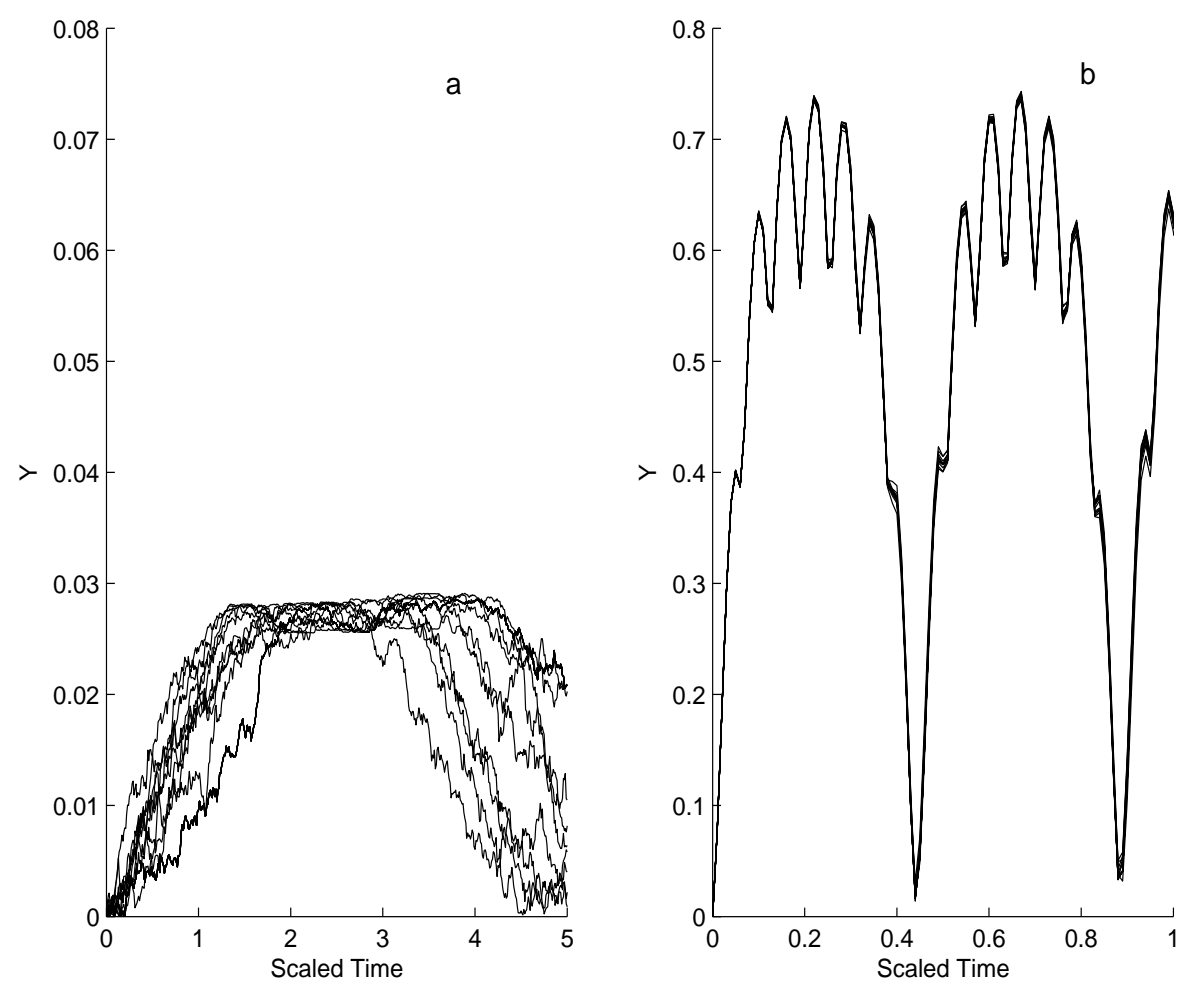

Figure 6: 